\title{
Retinal vessel structure measurement using spectral-domain optical coherence tomography
}

TH Rim ${ }^{1,4}$, YS Choi ${ }^{2,4}$, SS Kim¹, M-j Kang ${ }^{1}$, J Oh ${ }^{3}$, $\mathrm{S}$ Park ${ }^{3}$ and SH Byeon ${ }^{1}$

\begin{abstract}
Purpose To assess the reliability and validity of spectral-domain optical coherence tomography (SD-OCT) measurements of retinal vessel lumen diameters and wall thicknesses.

Methods SD-OCT was used to characterize the circular region around the optic disc of 40 eyes ( 20 subjects). The inner and outer sides (vitreal and choroidal sides) of the vessel wall and the luminal diameter were measured using intensity graphs.

Results Mean arterial and venous luminal diameters were $95.1 \pm 16.1$ and $132.6 \pm 17.8 \mu \mathrm{m}$, respectively. The wall thicknesses of inner and outer sides of the artery were $23.9 \pm 4.9$ and $21.2 \pm 3.5 \mu \mathrm{m}$, respectively. The wall thicknesses of the inner and outer sides of the vein were $20.7 \pm 4.2$ and $16.3 \pm 4.3 \mu \mathrm{m}$, respectively. There were significant differences between the inner and outer wall thicknesses in both the artery and vein $(P<0.01)$. Intra- and interobserver intraclass correlation coefficients (ICCs) for lumen measurements were $>0.95$, and for wall thicknesses were $>0.85$, except for the outer wall thickness measurements. The mean value of outer and inner wall thicknesses showed good reproducibility, with ICCs of $>0.85$.

Conclusion Intensity graph-assisted measurements using SD-OCT provided more objective information in finding boundaries of vessels. Luminal diameters and wall thicknesses obtained with OCT showed good overall reproducibility, with inner wall thicknesses being thicker, and with better reproducibility compared with outer wall thicknesses, where ICC values were the lowest among the inner wall thicknesses, mean thicknesses of inner and outer walls, and luminal diameters. When using SD-OCT measurements, caution is therefore advised
\end{abstract}

when using only the outer wall as representative of the wall thicknesses. Eye (2016) 30, 111-119; doi:10.1038/eye.2015.205; published online 23 October 2015

\section{Introduction}

The retinal vasculature, which can be associated with systemic diseases, ${ }^{1,2}$ can be directly visualized using noninvasive procedures. Historically, the first measurement of retinal vessel diameters was a year after the development of the ophthalmoscope. ${ }^{3}$ Currently, assessment of retinal vessel parameters using fundus photography, ${ }^{4,5}$ angiographic pictures, ${ }^{6}$ scanning laser Doppler flowmetry, ${ }^{7}$ Heidelberg retina angiograph images, ${ }^{8}$ or the combination of adaptive optics and optical coherence tomography $(\mathrm{OCT})^{9}$ remains under development. The standardized assessment of the arteriovenous ratio based on arterial and venous central equivalents was widely accepted and became the standard in epidemiologic studies. ${ }^{10}$ Recently, spectral-domain optical coherence tomography (SD-OCT) has been used for measuring the diameters of retinal blood vessels. Measurements of retinal wall thicknesses and diameters based on circular scans showed high reproducibility with intervisit, intra- and interobserver intraclass correlation coefficients (ICCs) of $>0.90 .{ }^{11}$ Other studies also reported high ICCs of $>0.95$ in measuring retinal outer diameters and retinal lumen diameters in zone B.

In the present study, we evaluated the reliability and validity of SD-OCT measurements of retinal vessel lumen diameters, with special emphasis upon the differences between inner (towards the vitreous) and outer (towards the retinal pigment epithelium) sides of the vessel wall.

\begin{abstract}
'Department of
Ophthalmology, Severance Hospital, Institute of Vision Research, Yonsei University College of Medicine, Seoul, South Korea
\end{abstract}

${ }^{2}$ Department of Radiology, Severance Hospital, Yonsei University College of Medicine, Seoul, South Korea

${ }^{3}$ Department of Cardiology, Severance Cardiovascular Hospital, Cardiovascular Research Institute, Yonsei University College of Medicine, Seoul, South Korea

Correspondence: SH Byeon, Department of Ophthalmology, Severance Hospital, Institute of Vision Research, Yonsei University College of Medicine, 50 Yonsei-ro, Seodaemun-gu, Seoul 120-752, South Korea

Tel: +82 22228 3570;

Fax: +82 23120541 .

E-mail: shbyeon@yuhs.ac

${ }^{4}$ These authors contributed equally to this work.

Received: 11 March 2015 Accepted in revised form: 16 August 2015 Published online: 23 October 2015 


\section{Materials and methods}

\section{Study population}

We retrospectively reviewed the medical records of 40 eyes of 20 patients characterized by SD-OCT circular scans in both eyes at Severance Hospital, Yonsei University College of Medicine, from January 2011 to July 2014. Criteria for inclusion were as follows: (1) patients $<40$ years of age, (2) patients without lens opacities, and (3) an SD-OCT image with resolution sufficient to measure the largest or second largest retinal vessel in both eyes. Criteria for exclusion were as follows: (1) patients with an SD-OCT image of low quality because of inflammation such as uveitis, high myopia, or lens opacity, and (2) patients with a pathologic disc, including papilledema, demyelinating nerve fiber, epiretinal membrane around the disc, or mass lesion. All the study protocols adhered to the tenets of the Declaration of Helsinki, and the study was approved by the Institutional Review Board/Ethics Committee. Written informed consent was waived.

\section{OCT imaging and measurements}

SD-OCT (Heidelberg Engineering Inc., Heidelberg, Germany) was performed using an internal fixation target. The focus of the camera was centered on the optic disc, and the fundus was visualized on the display. After obtaining OCT images of a circular scan, the vessels were dissected vertically using line volume of interest (VOI), and then an intensity graph was obtained using the Medical Image Processing, Analysis and Visualization (MIPAV), Version 7.0.1 (http:/ / mipav.cit.nih.gov; Center for Information Technology, NIH, Bethesda, MD, USA). Supplementary Figure 1 shows the detailed procedure for the measurements. The length was measured based on calculating the distance of point-to-point in the intensity graph. The scale factor was set according to the scale bar on the image, which was $200 \mu \mathrm{m}$ per 206 pixels (0.862 in, $7.27 \mathrm{~cm}$ using the MIPAV).

\section{Assessment of reproducibility}

The intraclass correlation coefficient (ICC) was used to determine reproducibility for selecting images sufficient to measure the largest or second largest artery and vein in each patient's OCT scan. To determine the reproducibility of vessel diameter measurements, a single retina specialist independently examined the (TH Rim) vessel diameters using the same OCT scan from 40 arteries and 40 veins from 40 eyes of 20 subjects on separate days. The first examiner (TH Rim) marked the same vessel, measured by another observer, a radiologist (YS Choi), who measured the vessel diameter on the same OCT scan from 40 arteries and 40 veins.

\section{Statistical analyses}

Vessel diameter parameters, including wall thickness, luminal diameter, and wall-to-lumen ratio, were determined. Mean wall thicknesses of the artery and vein were determined by location of the inner and outer sides of the vessel wall. Luminal diameter and wall-to-lumen ratios of the artery and vein were determined, and these parameters were compared with the right and left eye. Paired $t$-tests were used to compare quantitative data. To determine the reliability of the measurements, within-subject standard deviation was calculated by using the square root of the mean within-subject variance. Intraobserver repeatability and interobserver reproducibility were determined by ICCs. We classified ICCs of $\geq 0.81$ as almost perfect reliability, and ICCs of $\geq 0.61$ and $<0.8$ as substantial reliability. Bland and Altman plots were used to assess intraobserver repeatability and interobserver reproducibility. All statistical tests were two-sided at $95 \%$ confidence interval (CI), and a $P$-value $<0.05$ was considered statistically significant using the Stata/SE 13.1 software (StataCorp, College Station, TX, USA).

\section{Results}

Figure 1 shows cross-sectional images along the retinal arteriole. Figure 1a is an infrared photograph. The red arrow represents the location of the B-scan. Figure $1 \mathrm{~b}$ shows the cross-sectional B-scan of the arteriole in Figure 1a, and a magnified image of the B-scan is shown in Figure 1c. The alignment was not perfectly parallel to the vessel direction; however, the reflectivity of the inner wall was less continuous compared with the outer wall, and there was also an epiretinal membrane. The four red lines in Figure 1c represent the line VOI, and the intensity graphs from 1 to 4 were generated using the MIPAV. The highest peak (farthest left) in the intensity graph of line 1 represents the reflectivity of the epiretinal membrane, and the next highest peak represents the inner wall, which overlapped with the peak of the epiretinal membrane in the intensity graph of line 1 . It was therefore difficult to discriminate the inner wall in the intensity graph of line 2 and the outer wall in the intensity graph of line 3. Line 4 was the best line to discriminate the inner and outer walls; however, the junction of the lumen and inner wall was not sufficiently resolved for discrimination (arrow). An overall decreasing tendency of intensity from the outer to inner retina was observed in all intensity graphs.

Table 1 shows the parameters of the vessel walls. The thickness of the inner wall was thicker compared with the 

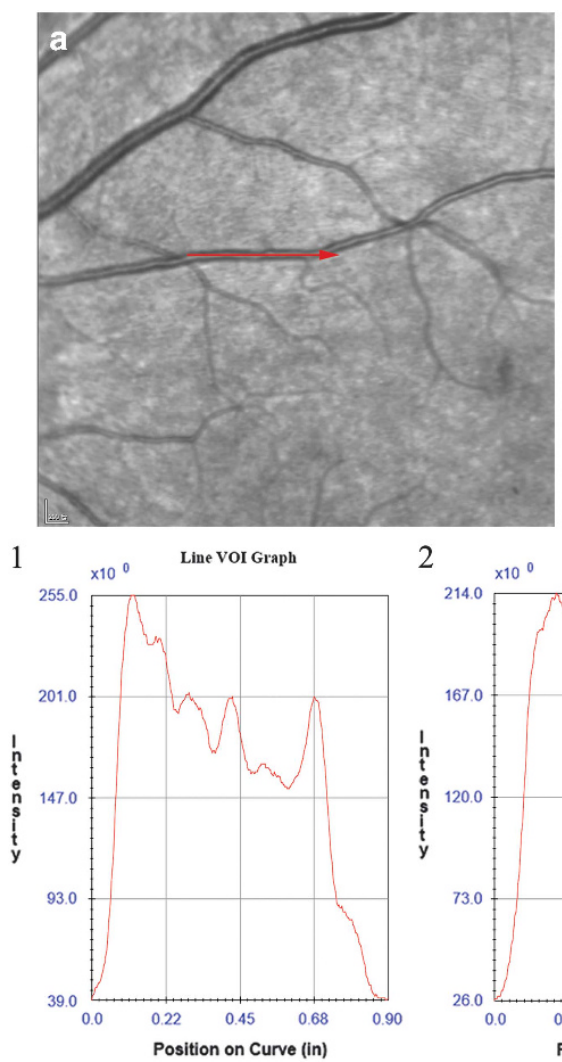

2

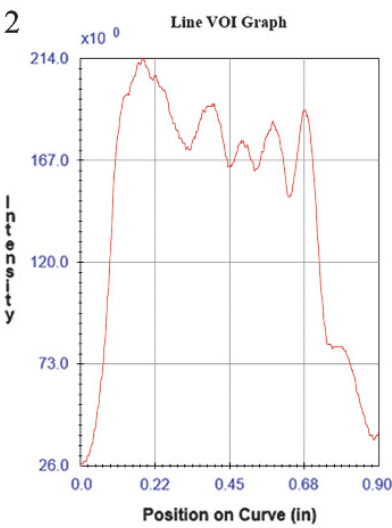

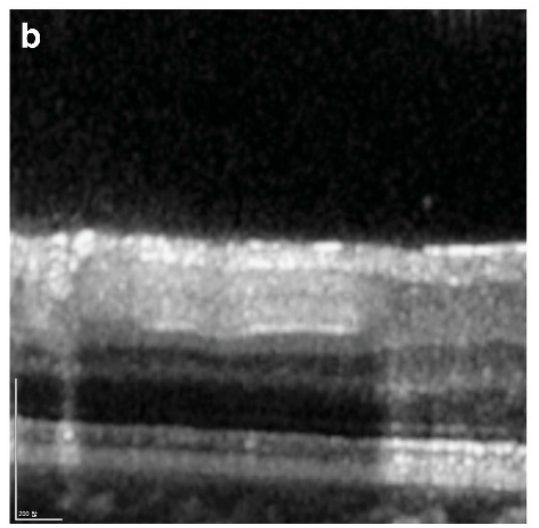

$3 \times 10^{0} \quad$ Line Vor Graph

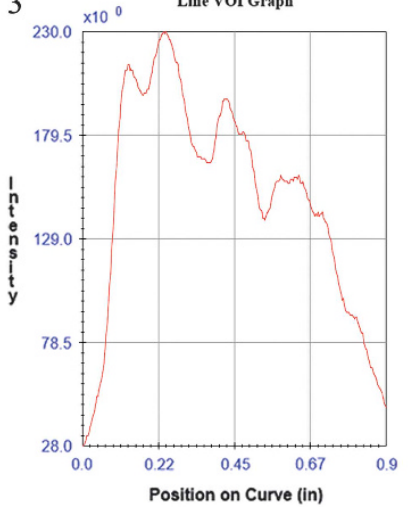

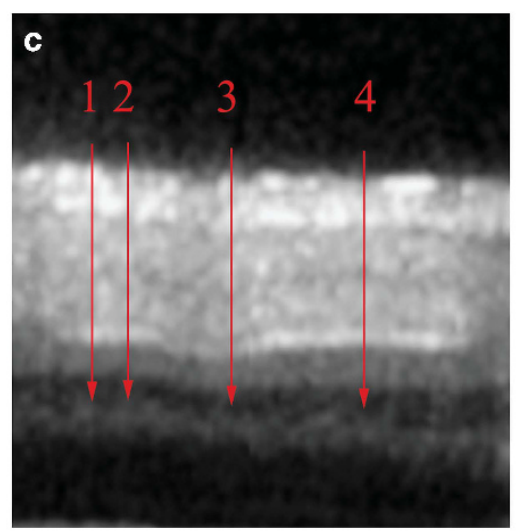

4

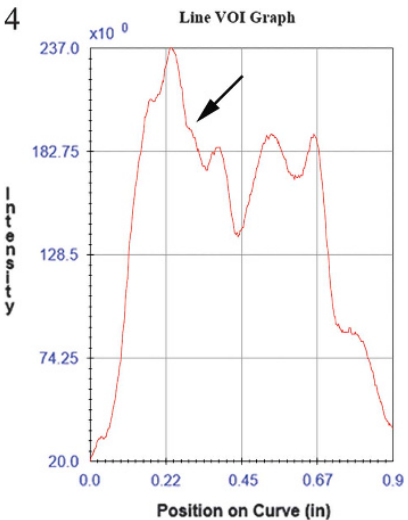

Figure 1 Retinal vessel image using SD-OCT and an intensity graph of the vertical section of vessels using MIPAV. The infrared photo (a) with the red arrow represents the location of the B-scan, with the cross-section of the corresponding arteriole (b), and the magnified image (c). The alignment was not perfectly parallel to the vessel direction; however, the reflectivity of the inner (towards the vitreous) wall is less continuous compared with the outer wall (towards the retinal pigment epithelium). The inner wall is difficult to discriminate in lines 1 and 2. The outer wall is difficult to discriminate in line 3, but better discriminated in line 4 . However, the margins of the inner wall and lumen are difficult to discriminate (arrow) (vertical-to-horizontal ratio 2:1).

outer wall in both the artery and vein $(P<0.01)$, and the gap between the inner wall and outer wall was $\sim 5 \mu \mathrm{m}$ for both the artery and vein. The luminal diameter of the largest or second largest vein was $132.6 \pm 17.8 \mu \mathrm{m}$ and that of the artery was $95.1 \pm 16.1 \mu \mathrm{m}$. The wall-to-lumen ratio was calculated separately using the inner, outer and mean of the inner and outer wall thicknesses. The wall-to-lumen ratio of the artery was $0.218 \pm 0.045$, and that of the vein was $0.117 \pm 0.027$, based on the inner wall. The difference of the wall-to-lumen ratio between the inner and outer walls was $\sim 0.05$ in both artery and vein. All parameters were the same between the right and left eyes.

Table 2 shows the intraobserver repeatability of measurements of the retinal lumen, inner and outer wall thicknesses, and mean of the inner and outer wall thicknesses of the artery and vein. The intraobserver ICCs for the parameters including lumen and wall thicknesses were between 0.850 and 0.980 , which represented very good measurement reliability, with the exception of the arteriolar outer wall thickness of 0.712 .

Table 3 shows the interobserver reproducibility of measurements. The interobserver reproducibility was slightly lower compared with the intraobserver reproducibility. The interobserver ICCs were between 0.841-0.973, which represented very good reliability of measurements, with the exception of the arteriolar outer wall thickness of 0.681 .

Figures 2 and 3 show the Bland-Atman plots, which show good agreement of both intra- and interobserver measurements of all parameters. The mean difference between two examinations by one observer ranged from -0.025 to 1.281 , and the mean difference between two examinations by different observers ranged from -0.296 to 0.542 .

\section{Discussion}

In the present study, we demonstrated that an intensity graph-assisted vessel measurement using SD-OCT provided an accurate determination of the boundaries of vessel structures. Based on ICCs and the Bland-Atman plot, we confirmed the reliability and validity of SD-OCT measurements of the retinal vessel wall thickness and lumen of both the artery and vein. Furthermore, based on 
Table 1 Retinal arterial and venous diameter measurements by one observer using SD-OCT

\begin{tabular}{|c|c|c|c|}
\hline & $\begin{array}{c}\text { Mean } \pm S D \\
\text { Inner }(\mathrm{n}=40)\end{array}$ & $\begin{array}{c}\text { Mean } \pm S D \\
\text { Outer }(\mathrm{n}=40)\end{array}$ & P-value \\
\hline \multicolumn{4}{|l|}{ Wall thickness by location } \\
\hline Wall thickness, artery & $23.9 \pm 4.9$ & $21.2 \pm 3.5$ & $<0.01$ \\
\hline \multirow[t]{2}{*}{ Wall thickness, vein } & $20.7 \pm 4.2$ & $16.3 \pm 4.3$ & $<0.01$ \\
\hline & Artery $(n=40)$ & $\operatorname{Vein}(\mathrm{n}=40)$ & \\
\hline \multicolumn{4}{|l|}{ Wall-to-lumen ratio in artery and vein } \\
\hline Luminal diameter & $95.1 \pm 16.1$ & $132.6 \pm 17.8$ & $<0.01$ \\
\hline Wall-to-lumen ratio, inner & $0.258 \pm 0.061$ & $0.158 \pm 0.034$ & $<0.01$ \\
\hline Wall-to-Lumen ratio, outer & $0.229 \pm 0.051$ & $0.125 \pm 0.038$ & $<0.01$ \\
\hline Wall-to-lumen ratio, mean & $0.243 \pm 0.048$ & $0.142 \pm 0.028$ & $<0.01$ \\
\hline \multicolumn{4}{|l|}{ Parameters by right/left eye } \\
\hline \multicolumn{4}{|l|}{ Artery } \\
\hline Luminal diameter & $94.4 \pm 15.2$ & $95.9 \pm 17.2$ & 0.78 \\
\hline Wall thickness, inner & $25.6 \pm 4.1$ & $24.3 \pm 4.7$ & 0.35 \\
\hline Wall thickness, outer & $20.1 \pm 2.8$ & $20.2 \pm 3.2$ & 0.94 \\
\hline Wall-to-Lumen ratio, inner & $0.274 \pm 0.048$ & $0.242 \pm 0.069$ & 0.10 \\
\hline Wall-to-Lumen ratio, outer & $0.219 \pm 0.039$ & $0.239 \pm 0.061$ & 0.21 \\
\hline \multicolumn{4}{|l|}{ Vein } \\
\hline Luminal diameter & $135.3 \pm 17.1$ & $129.9 \pm 18.6$ & 0.35 \\
\hline Wall thickness, inner & $25.6 \pm 4.1$ & $21.1 \pm 4.0$ & 0.33 \\
\hline Wall thickness, outer & $16.2 \pm 3.6$ & $14.5 \pm 2.9$ & 0.11 \\
\hline Wall-to-lumen ratio, inner & $0.158 \pm 0.033$ & $0.158 \pm 0.036$ & 0.96 \\
\hline Wall-to-lumen ratio, outer & $0.131 \pm 0.045$ & $0.119 \pm 0.031$ & 0.33 \\
\hline
\end{tabular}

Abbreviations: SD-OCT, spectral-domain optical coherence tomography; SD, standard deviation.

Table 2 Intraobserver repeatability of retinal vessel diameter measurements by optical coherence tomography

\begin{tabular}{|c|c|c|c|c|}
\hline & $S w(\mu m)$ & Repeatability coefficient $(\mu \mathrm{m})$ & & CI) \\
\hline \multicolumn{5}{|l|}{ Artery } \\
\hline Lumen & 1.92 & 5.31 & 0.986 & $0.977-0.995$ \\
\hline Wall thickness, inner & 1.49 & 4.14 & 0.876 & $0.804-0.949$ \\
\hline Wall thickness, outer & 1.66 & 4.60 & 0.712 & $0.558-0.866$ \\
\hline Wall thickness, mean & 1.28 & 3.54 & 0.854 & $0.769-0.938$ \\
\hline \multicolumn{5}{|l|}{ Vein } \\
\hline Lumen & 2.63 & 7.29 & 0.977 & $0.963-0.991$ \\
\hline Wall thickness, inner & 1.30 & 3.61 & 0.871 & $0.796-0.946$ \\
\hline Wall thickness, outer & 1.26 & 3.50 & 0.850 & $0.763-0.937$ \\
\hline Wall thickness, mean & 1.09 & 3.01 & 0.869 & $0.792-0.945$ \\
\hline
\end{tabular}

Abbreviations: CI, confidence interval; ICC, intraclass correlation coefficient; Sw, within-subject standard deviation.

the intensity graph using the MIPAV, the thicknesses of the inner and outer walls were different.

Measurement of vessels using SD-OCT is currently being developed as a methodology to accurately determine vessel parameters. Normal retinal vessels appear as oval-shaped configurations with four distinct hyperreflectivities, with a double ' $\mathrm{C}$ ' pattern, which may result from blood flow within retinal vessels, based on experimental animals and the glass tube model. ${ }^{11-13}$ Two previous reports differed with respect to the measure points in infrared images. Muraoka et al ${ }^{11}$ used zone A and reported that the OCT beam was projected perpendicular to the retinal surface in a circular scan, with vertical sections of all major retinal vessels reliably obtained with a single scan. However, Zhu et al ${ }^{14}$ reported that SD-OCT measurements of vessels in zone B were more accurate compared with in zone A. Currently, discriminating the junction of the wall and lumen, or wall 
Table 3 Interobserver reproducibility of retinal vessel diameter measurements by optical coherence tomography

\begin{tabular}{|c|c|c|c|c|}
\hline & $S w(\mu m)$ & Repeatability coefficient $(\mu \mathrm{m})$ & & CI) \\
\hline \multicolumn{5}{|l|}{ Artery } \\
\hline Lumen & 2.62 & 7.25 & 0.973 & $0.956-0.989$ \\
\hline Wall thickness, inner & 1.50 & 4.15 & 0.874 & $0.801-0.948$ \\
\hline Wall thickness, outer & 1.73 & 4.81 & 0.681 & $0.514-0.848$ \\
\hline Wall thickness, mean & 1.27 & 3.51 & 0.852 & $0.766-0.937$ \\
\hline \multicolumn{5}{|l|}{ Vein } \\
\hline Lumen & 3.94 & 10.91 & 0.950 & $0.920-0.981$ \\
\hline Wall thickness, inner & 1.34 & 3.72 & 0.859 & $0.778-0.941$ \\
\hline Wall thickness, outer & 1.33 & 3.69 & 0.841 & $0.750-0.932$ \\
\hline Wall thickness, mean & 1.12 & 3.09 & 0.865 & $0.786-0.943$ \\
\hline
\end{tabular}

Abbreviations: Sw, within-subject standard deviation; ICC, intraclass correlation coefficient; CI, confidence interval.

\section{a}

$2 / 40=5.00 \%$ outside the limits of agreement Mean difference -0.025 $95 \%$ limits of agreement $(-5.404,5.355)$

15 Averages lie between 64.335 and 138.259

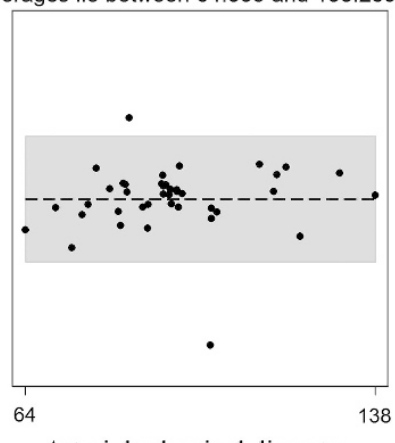

Arteriolar luminal diameter

d $\quad 1 / 40=2.50 \%$ outside the limits of agreement $1 / 40=2.50 \%$ outside
Mean difference 1.281

$95 \%$ limits of agreement $(-5.655,8.216)$

15 verages lie between 93.022 and 166.818

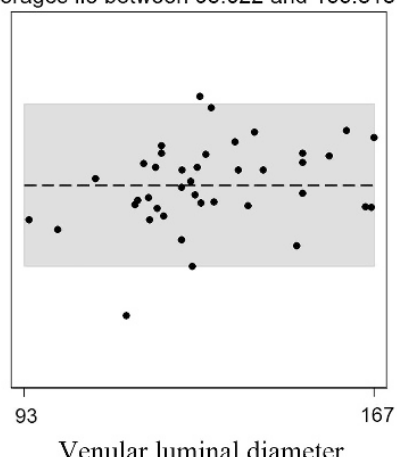

b

$1 / 40=2.50 \%$ outside the limits of agreement Mean difference 0.463 $95 \%$ limits of agreement $(-4.109,5.035)$ Averages lie between 13.196 and 25.396

15

$-$

13

Outer arteriolar wall thickness

e $2 / 40=5.00 \%$ outside the limits of agreement Mean difference 0.482 $95 \%$ limits of agreement $(-2.931,3.895)$

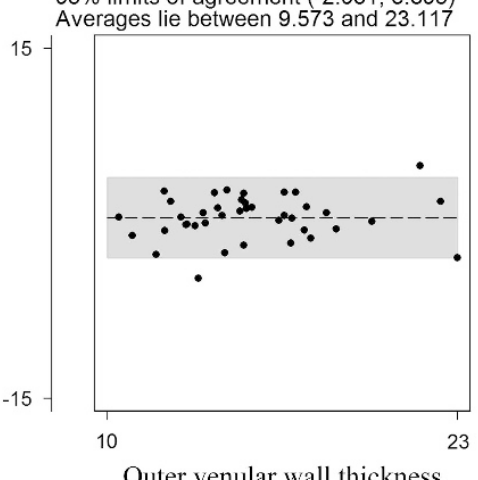

C

$2 / 40=5.00 \%$ outside the limits of agreement Mean difference 0.785 $95 \%$ limits of agreement $(-3.107,4.676)$

15 Averages lie between 16.218 and 33.956

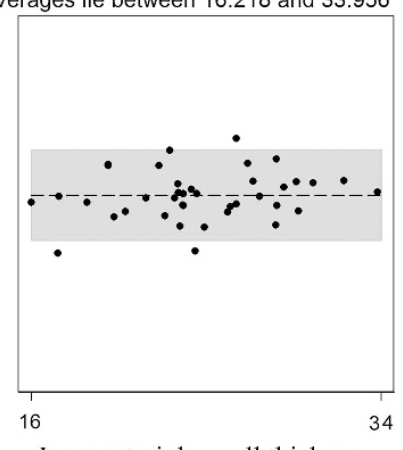

Inner arteriolar wall thickness

f $2 / 40=5.00 \%$ outside the limits of agreement

Mean difference 0.608 limits of agreement $(-2.843,4.059)$ $95 \%$ limits of agreement $(-2.843,4.059)$
Averages lie between 14.525 and 30.000

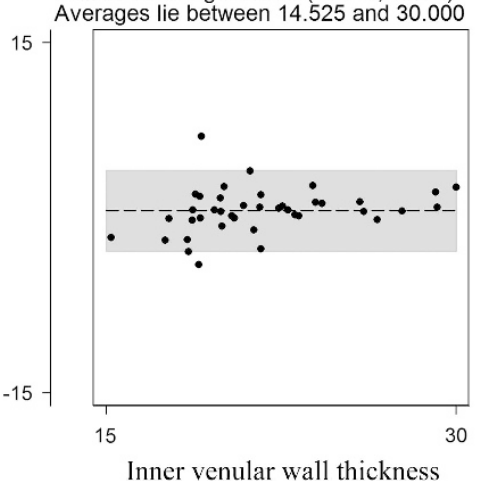

Figure 2 Agreement between two examinations by one observer using Bland-Altman analyses. The Bland-Altman plot shows differences in arteriolar (a) and venular (d) luminal diameters, outer arteriolar (b) and venular (e) diameters, and inner arteriolar (c) and venular ( $\mathrm{f}$ ) diameters between two examinations performed by the same observer.

and adjacent structure, may be more important compared with other preceding issues. To overcome the variability of measurements by each examiner, we employed an intensity graph using line VOI in the MIPAV. As shown in Figure 1 and 4, the intensity graph provided more objective information of the boundary, which has been elusive in the original SD-OCT images, and which may depend on individual examiners. Furthermore, we suggest that the most accurate conclusions can be obtained by combining information from both the intensity graph and the SD-OCT circular B-scan images.

Figure 1 and 4 were used differently to assess the vessel; however, we could obtain partial information from Figure 1. As shown in this figure, the degree of 

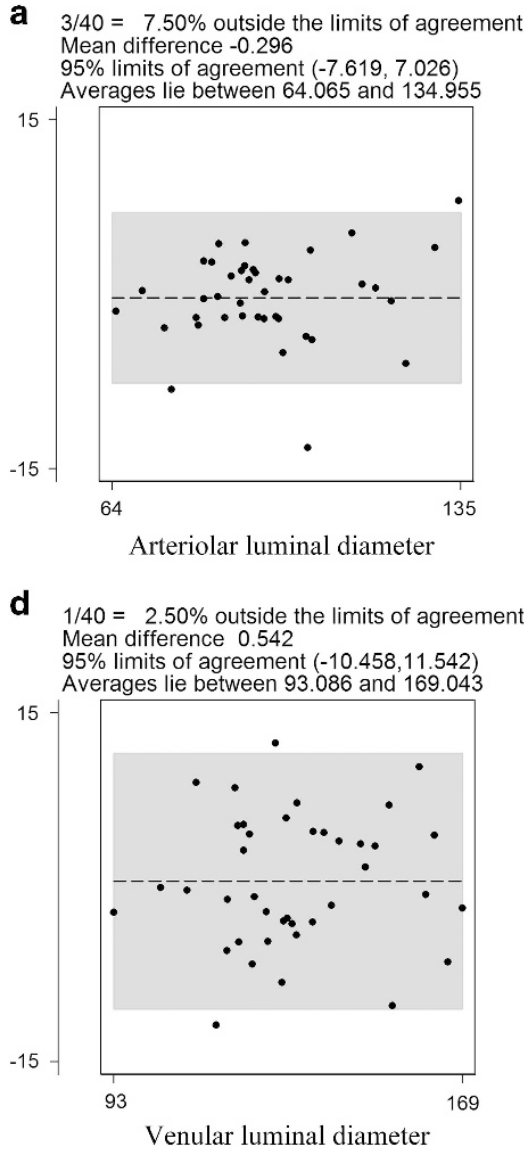

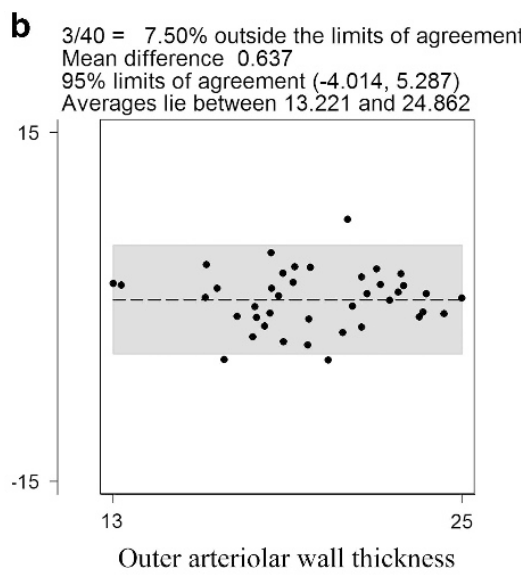

e $1 / 40=2.50 \%$ outside the limits of agreement

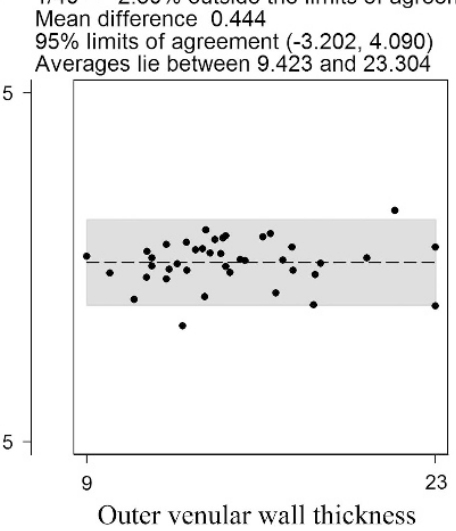

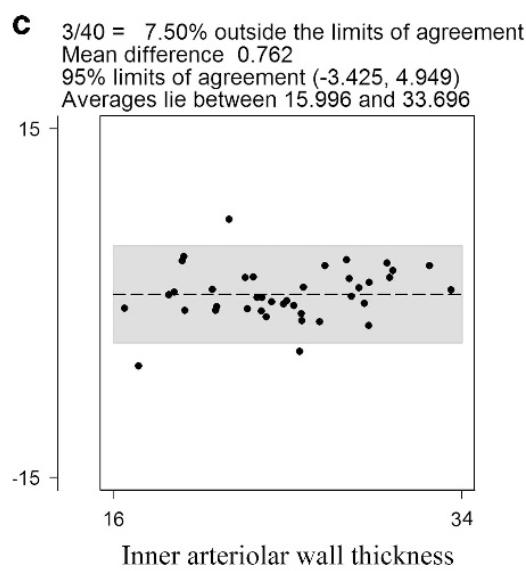

$\mathbf{f}$

$2 / 40=5.00 \%$ outside the limits of agreement Mean difference 0.551

$95 \%$ limits of agreement $(-3.056,4.158)$

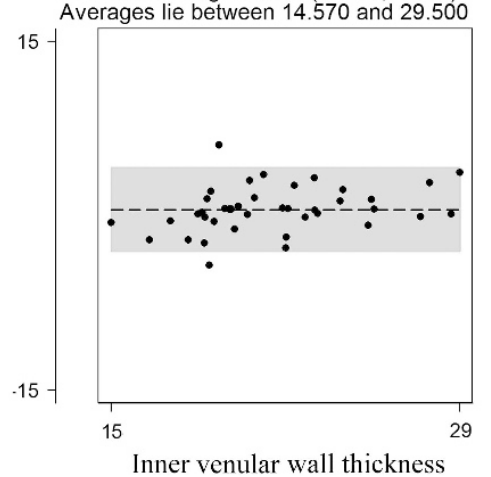

Figure 3 Agreement between two examinations by one observer using Bland-Altman analyses. The Bland-Altman plot shows differences in arteriolar (a) and venular (d) luminal diameters, outer arteriolar (b) and venular (e) diameters, and inner arteriolar (c) and venular (f) diameters between two examinations performed by two observers.

hyperreflectivity of inner and outer walls was different; the inner wall was less continuous compared with the outer wall. Vessel measurements using SD-OCT are sometimes difficult because the hyper-reflective tissues around the vessel, such as the epiretinal membrane, can confuse discrimination of the anterior surface of the inner wall (line 1 in Figure 1), and the inner or outer wall reflectivity is sometimes difficult to find (lines 2 and 3, respectively, in Figure 1), or the margin between the lumen and wall is sometimes unclear (arrow in line 4 in Figure 1 denotes margin). The different discontinuities of hyperreflectivity between the inner and outer walls may suggest different situations in the circular scan. In the circular scan of Figure 4, there are two circular hyperreflections at the inner wall in line 1 , and the small circle designated by the arrow in the magnified image in Figure 1a may reflect the inner wall peak. The inner wall in line 2 looks like the alphabet letter ' $U$ ', which could contribute to inaccuracies in measurements of inner wall thicknesses. However, the intensity graph of line 2 shows a clearer peak of the inner wall. The best cross-section and intensity graph is line 3 in Figure 4, which clearly discriminates the inner and outer walls, as well as the luminal diameter. The intensity graph in Figure 4 shows that the inner wall thickness was thicker compared with the outer wall thickness, which may have resulted from a decrease of light penetration, with a resultant decrease in reflectivity. A slight decrease of peak height corresponding to the outer wall compared with the inner wall is shown in line 1. However, in lines 2 and 3, the intensity was decreasing with depth. Different sizes of the inner and outer walls were also reported in previous studies. ${ }^{11,14}$ The inner walls were thicker compared with the outer walls, with $75 \%$ in arteries (30/40, data not shown) and $82.5 \%$ in veins (33/40, data not shown). In general, the inner wall was more intense and thicker compared with the outer wall because of decreases in light intensity at increasing distances.

Previous studies involving direct measurements of vessel diameters using the Image J software (National Institutes of Health, Bethesda, MD, USA) reported high repeatability and reproducibility. ${ }^{11,14}$ In our study, the intra- and 

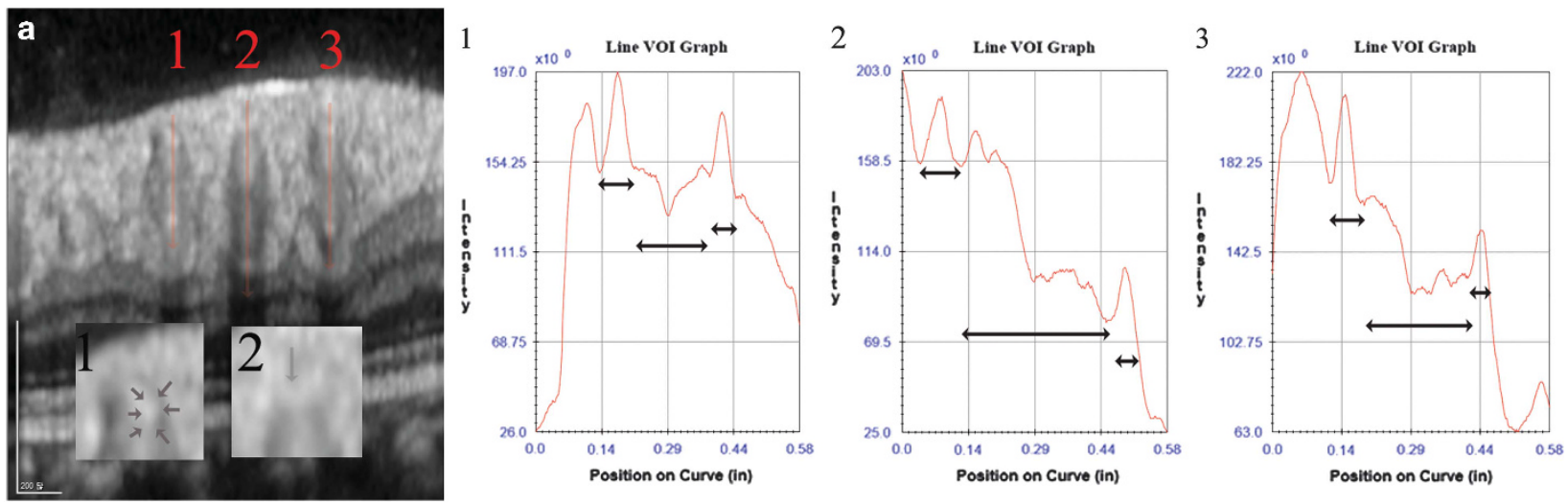

Figure 4 A circular scan of a vessel cross-section. (a) A cross-section of the artery (vessel 1), vein (vessel 2), and artery (vessel 3). There was dual circular reflectivity at the inner wall of vessel 1, and in the magnified image of the inner wall of vessel 1 (bottom left), the gray arrow represents the smaller circular refractivity in the inner wall. The shape of the inner wall of vessel 2 resembles the alphabet letter ' $U$ ', and the gray arrow may represent the boundary of reflectivity resulting from the inner wall in the magnified image of vessel 2 (bottom right). The double arrow at the left in the intensity graph represents the inner wall thickness. The double arrow at the center and right in the intensity graph represents the luminal diameter and outer wall thickness, respectively. The intensity graph of lines 1 and 2 show that the length of the inner wall thickness is thicker compared with the outer wall thickness. However, the thickness of the wall is similar in vessel 3 based on the intensity graph.

interobserver ICCs for OCT vessel diameter measurements were also highly repeatable and reproducible, especially in measuring the luminal diameter, the ICCs were $>0.90$. Other parameter measurements also had relatively good ICCs of $>0.80$. However, the measurement of arterial wall thicknesses of the outer wall was relatively inaccurate, with an intraobserver ICC of 0.712 and an interobserver ICC of 0.681 . Repeatability was relatively low in measuring the outer wall compared with the inner wall, or lumen, because the reflectivity peak was relatively small and there may have been a relatively large change in length from the small change of line VOI location. However, the inaccuracy of the outer wall could be overcome by using the mean value of the inner and outer wall thicknesses, with ICCs $\geq 0.85$.

There was a difference between the inner and outer walls. The inner wall thicknesses obtained with OCT were thicker and more reproducible compared with the outer wall thicknesses. Outer wall thicknesses had fair reproducibility; however, caution is advised when using the outer wall alone as a standard for wall thickness in SD-OCT measurements because of relatively poor repeatability compared with measurements of the inner walls or lumen diameters. A recent study reported almost perfect reproducibility of retinal vessel diameter measurements based on direct measurement using the Image J software (National Institutes of Health), with ICCs ranging from 0.944 to 0.982 in one study, ${ }^{11}$ and 0.968 to 0.981 in another study. ${ }^{14}$ Although our ICCs were lower compared with previous reports, they still represented the usefulness of measuring diameters using SD-OCT, and represented the value of using a standard vessel parameter with high validity. The Bland-Altman plot (Figure 2 and 3) shows that the interclass 95\% limits of agreement width for intraobserver repeatability were greater than that for interobserver reproducibility. In terms of expertise in ophthalmology specialties, interobserver reproducibility was calculated based on two examinations performed by two observers (T Rim and YS Choi). The first observer was a retinal specialist, and the second observer was a neuroradiologist. Although the radiologist was familiar with black and white test results, it was the first time the neuroradiologist read the retinal SD-OCT images. Thus, if proper training is available in future studies, it is possible that an ophthalmologist may not be necessary in interpreting measurements of vessel diameters.

We believe a circular scan is not sufficient to measure all vessel diameters. However, a circular scan can provide at least one or more high-resolution images. In most cases, we can measure the diameter of the largest artery and vein using a clear image. However, sometimes, it is impossible to measure the largest artery or vein because of crossing of the artery and vein, or because of an inaccurate cross-section that is not perpendicular to the circle scan. Because of these reasons, we chose the largest or second largest artery and vein, which had sufficient resolution for measurements. As mentioned in a previous report, ${ }^{14}$ measurement in zone B will increase the test time and may cause the patient to become too fatigued to cooperate. For this reason, the circular scan mode is already part of a commercial SD-OCT, thus the problem of low-quality circular scan can be overcome by selecting a few vessels and by increasing the number of scans.

In terms of the real length, errors can arise from the individual properties of the eye such as lens opacity and 
refractive error, or from the imaging system involving fundus photographs, infrared images, image processing, magnification scale and vessel diameter changes during the cardiac cycle. These factors result in difficulties in comparing diameter measurements using the different methods. Thus, our study only used SD-OCT

measurements. Histological studies reported that arteries adjacent to the optic disc had a luminal diameter of $\sim 0.1 \mathrm{~mm}$, their walls were $\sim 18 \mu \mathrm{m}$, and the principal branches of the central retinal vein had luminal diameters of $\sim 0.2 \mathrm{~mm}$. In our study, the luminal diameter was $95.1 \pm 16.1 \mu \mathrm{m}$ for arteries and $132.6 \pm 17.8 \mu \mathrm{m}$ for veins. Previous studies using SD-OCT measurements reported the arterial and venous luminal diameters (inner diameters) were $87.8 \pm 9.4$ and $113.7 \pm 12.5$, respectively, which were shorter compared with our results. The arteriolar and venous wall thicknesses using the outer wall were $20.2 \pm 3.0 \mu \mathrm{m}$ and $15.4 \pm 3.3 \mu \mathrm{m}$, respectively. Previous studies reported that arterial and venous wall thicknesses averaged $17.4 \pm 2.4 \mu \mathrm{m}$, and $13.7 \pm 2.1 \mu \mathrm{m}$, respectively, which were also thicker compared with our wall thicknesses, even if we used the outer wall. The previous study selected the four largest retinal arteries and veins, and mean values were calculated from these vessels. In our study, we chose only one vessel of the largest or second largest artery and vein, depending on their image qualities. Note that upper arterial wall thickness was $25.0 \pm 3.5 \mu \mathrm{m}$ in our study, which was much larger compared with the histological measurements.

The clinical significance of retinal vessels as a cardiovascular risk factor has been generally accepted. ${ }^{15-20}$ Therefore, the assessment of vessel diameters is highly desirable. OCT technologies have greatly improved; however, future OCT technologies such as the combination of adaptive optics and OCT will have a higher resolution that will enable observation at the cellular level in the near future. ${ }^{21}$ Vessel measurement via fundus photography also has advantages, including simple and semiautomated measurement. However, the accuracy of fundus photography may be lower compared with that of OCT.22 Our new methodology involving intensity graph-assisted vessel measurements using SDOCT may provide a new direction for future software development, which may in turn lead to automated vessel wall quantification on intensity graphs from SD-OCT scans. Thus, SD-OCT itself could be used as an alternative, noninvasive tool for assessing retinal vessels as a cardiovascular risk factor.

The strength of the present study was that the intensity graph-assisted measurements of vessel parameters could be a more objective measurement compared with a direct measurement using the Image J software (National Institutes of Health). The major limitation of our study was the lack of a comparison with previously validated vessel diameter measurements such as 'IVAN'. It is essential to increase the reliability of measurements compared with conventional methods, but it was sometimes difficult to obtain complete information via the circular scan of OCT images of the top six arteries or veins by size. Thus, further studies are needed involving comparisons of arteriovenous ratios among selected subjects using high-quality images. The intereye correlation may have duplicated the effect on the value of the vessel parameter; therefore, the parameters of the vessel wall may have also affected by the participant factor. In this case, if one participant had a retinal vessel with a relatively large diameter, this could affect the mean value of retinal vessel parameter by a factor of two. The four major vessels from the disc in one eye showed different characteristics when measuring parameters. Therefore, each vessel may be able to be applied independently on a limited basis when accessing the reliability based on ICC. In addition, our study was comprised of a relatively small sample size, which resulted in a reduced representation of parameters such as arterial diameter.

In conclusion, in the present study we have introduced new methodology involving intensity graph-assisted vessel measurements using SD-OCT, which provided more objective information in identifying vessel structure boundaries, compared with direct measurements using the Image J software (National Institutes of Health). Furthermore, measurements of vessel parameters, including the lumen and wall using SD-OCT, showed good reliability. However, reproducibility of outer wall thicknesses was the lowest compared with other vessel parameters, including lumen diameter, inner wall thickness, and the mean value of the inner and outer wall thicknesses. Therefore, an average value of the inner wall and outer walls, or the inner wall alone, were best in assessing the vessel, but caution is advised in using only the outer wall alone as representative of wall thickness.

\section{Summary}

\section{What was known before}

- SD-OCT has been used for measuring the diameters of retinal blood vessels.

- Measurements of retinal wall thicknesses and diameters based on SD-OCT showed high reproducibility with ICCs of $>0.90$.

What this study adds

- Intensity graph-assisted measurements of vessels based on SD-OCT circular scans around the optic disc facilitated easy identification of the boundary of the vessel walls.

- There were significant differences between the inner and outer wall thicknesses in both the artery and vein.

- Measurements of vessel parameters, including the lumen and wall thicknesses using SD-OCT, showed good reproducibility. 


\section{Conflict of interest}

The authors declare no conflict of interest.

\section{Acknowledgements}

This work was supported by a grant from the Korean Health Technology R\&D Project, Ministry of Health \& Welfare, Republic of Korea (HI13C0715).

\section{References}

1 Ong YT, Wong TY, Klein R, Klein BE, Mitchell P, Sharrett AR et al. Hypertensive retinopathy and risk of stroke. Hypertension 2013; 62(4): 706-711.

2 Wong TY, Mitchell P. Hypertensive retinopathy. N Engl J Med 2004; 351(22): 2310-2317.

3 Mikuni M. Eine Methode zur Messung der Netzhautgefäßweite. Klin Monatsbl Augenheilkd 1959; 135: 205-211.

4 Rassam SM, Patel V, Brinchmann-Hansen O, Engvold O, Kohner EM. Accurate vessel width measurement from fundus photographs: a new concept. Br J Ophthalmol 1994; 78(1): 24-29.

5 Bracher D, Dozzi M, Lotmar W. Measurement of vessel width on fundus photographs. Albrecht Von Graefes Arch Klin Exp Ophthalmol 1979; 211(1): 35-48.

6 Sandor T, Rhie FH, Soeldner JS, Gleason RE, Rand LI. Reproducibility of the densitometric analysis of fluorescein angiograms. Int J Biomed Comput 1981; 12(5): 401-418.

7 Raff U, Harazny JM, Titze SI, Schmidt BM, Michelson G, Schmieder RE. Salt intake determines retinal arteriolar structure in treatment resistant hypertension independent of blood pressure. Atherosclerosis 2012; 222(1): 235-240.

8 Formaz F, Riva CE, Geiser M. Diffuse luminance flicker increases retinal vessel diameter in humans. Curr Eye Res 1997; 16(12): 1252-1257.

9 Miller DT, Kocaoglu OP, Wang Q, Lee S. Adaptive optics and the eye (super resolution OCT). Eye 2011; 25(3): 321-330.

10 Hubbard LD, Brothers RJ, King WN, Clegg LX, Klein R, Cooper LS et al. Methods for evaluation of retinal microvascular abnormalities associated with hypertension/ sclerosis in the Atherosclerosis Risk in Communities Study. Ophthalmology 1999; 106(12): 2269-2280.

11 Muraoka Y, Tsujikawa A, Kumagai K, Akiba M, Ogino K, Murakami T et al. Age- and hypertension-dependent changes in retinal vessel diameter and wall thickness: an optical coherence tomography study. Am J Ophthalmol 2013; 156(4): 706-714.

12 Muraoka Y, Tsujikawa A, Murakami T, Ogino K, Kumagai K, Miyamoto K et al. Morphologic and functional changes in retinal vessels associated with branch retinal vein occlusion. Ophthalmology 2013; 120(1): 91-99.

13 Cimalla P, Walther J, Mittasch M, Koch E. Shear flowinduced optical inhomogeneity of blood assessed in vivo and in vitro by spectral domain optical coherence tomography in the 1.3 mum wavelength range. J Biomed Opt 2011; 16(11): 116020.

14 Zhu TP, Tong YH, Zhan HJ, Ma J. Update on retinal vessel structure measurement with spectral-domain optical coherence tomography. Microvasc Res 2014; 95: 7-14.

15 Graham EM. The investigation of patients with retinal vascular occlusion. Eye 1990; 4(Part 3): 464-468.

16 Hayreh SS. Duke-elder lecture. Systemic arterial blood pressure and the eye. Eye 1996; 10(Part 1): 5-28.

17 Leung H, Wang JJ, Rochtchina E, Wong TY, Klein R, Mitchell P. Dyslipidaemia and microvascular disease in the retina. Eye 2005; 19(8): 861-868.

18 Varma DD, Cugati S, Lee AW, Chen CS. A review of central retinal artery occlusion: clinical presentation and management. Eye 2013; 27(6): 688-697.

19 Rim TH, Kim DW, Han JS, Chung EJ. Retinal vein occlusion and the risk of stroke development: a 9-year nationwide population-based study. Ophthalmology 2015; 122(6): 1187-1194.

20 Wang J, Zhao M, Li SJ, Wang DZ. Retinal artery lesions and long-term outcome in Chinese patients with acute coronary syndrome. Eye 2015; 29(5): 643-648.

21 Keane PA, Sadda SR. Imaging chorioretinal vascular disease. Eye 2010; 24(3): 422-427.

22 Drexler W, Morgner U, Ghanta RK, Kartner FX, Schuman JS, Fujimoto JG. Ultrahigh-resolution ophthalmic optical coherence tomography. Nat Med 2001; 7(4): 502-507.

Supplementary Information accompanies this paper on Eye website (http://www.nature.com/eye) 